\title{
Analysis of Electrical Microgrids and Associated Technologies
}

\author{
J.I. San Martin ${ }^{1}$, F.J. Asensio ${ }^{1}$, I. Zamora ${ }^{2}$, G. Saldaña ${ }^{1}$, O. Oñederra ${ }^{2}$, I.J. Oleagordía ${ }^{2}$ \\ Department of Electrical Engineering - University of the Basque Country (UPV/EHU)
}

\author{
${ }^{1}$ Engineering School of Gipuzkoa, \\ Avda. Otaola, 29, 20600 Eibar (Spain) \\ e_mail: joseignacio.sanmartin@ehu.eus
}

\author{
${ }^{2}$ Engineering School of Bilbao \\ Alda. Urquijo s/n, 48013 Bilbao (Spain) \\ e_mail: inmaculada.zamora@ehu.eus
}

\begin{abstract}
This paper focuses on the most relevant aspects on the energy generation and storage technologies integration into electrical microgrids (MGs) structures. In this sense, the most important characteristics associated with electric generation resources proposed for this type of grids are reviewed. Additionally, energy storage technologies are analysed for the most appropriate integration into MGs. Finally, the manuscript reviews the most outstanding MGs developed around the world that include the integration of the most advanced generation and storage technologies.
\end{abstract}

\section{Key words}

Distributed Generation, Microgrids, Generation technologies, Storage Technologies, Distributed Technologies applications.

\section{Introduction}

Currently, there is a trend towards decentralization, decarbonisation and democratization of the electrical systems [1]. This trend, also known as the "three D's", seeks to replace obsolete infrastructures, to improve the reliability and quality of electricity supply, to provide energy to remote areas of difficult access and to reduce harmful emissions. In this sense, microgrids (MG) behave like architectures of electrical networks that allow access to electrical and thermal energy to broad sectors of the population, from residential areas of cities to rural areas of difficult access.

The concept of MG as a distributed small-scale generation in DC was introduced by Thomas Edison at the end of the 19 th century. The growing demand caused the centralization and consolidation of large generating units and long power transport lines [2]. From the 1920s to the 1970 s, centralized generation was promoted based on the ability to extract energy from generation resources which were far from the consumption location, thus promoting the development of the current power grid [3,4]. However, environmental concerns, Distributed Energy Resource (DER) technologies, and the economic risks inherent in the construction of massive generation facilities and transmission infrastructures are causing electric utilities to gradually transform from large and centralized to small and decentralized infrastructures over time. A network architecture that is able to manage the power generation and to supply subsections of the network that could be automatically isolated from a larger network is one of solutions that is being promoted. This way, subsections could provide critical services even when the main network fails. This approach was called "Microgrid" [2,4].

A MG is a small-scale power grid that consists of distributed generation (DG) units, energy storage systems (ESS), and electrical and thermal loads that operate as a single control unit at the distribution voltage level. The MGs can operate connected to the main grid or in an isolated way [5-9]. In general, under normal operation, the MG will work connected to the network, but in case of any disturbance occurs in the main network, the MG will disconnect quickly from it and will continue operating in island mode [10].

MGs must have their own control to guarantee the correct operation and coordination of the different DERs. In general, a Microgrid Central Controller (MGCC) is needed to manage the operation within the $\mathrm{MG}$, the energy flows and the interconnection with the main network. In addition, all devices of the MG need to communicate with the MGCC. Traditionally, this control is carried out through a three-level hierarchical scheme [11,12]: Distribution Management System (DMS) or tertiary control, Microgrid Central Controller (MGGC) or secondary control and Load Control (LC) or primary control $[13,14,15,16]$. Fig. 1 shows a typical example of an electrical MG in which the DMS, MGGC and LCs can be shown.

The primary control operates in the time range from milliseconds to minutes and reacts to the transient dynamics of the DERs and the system, responding to any instantaneous deviation in the voltage or frequency of the system. 


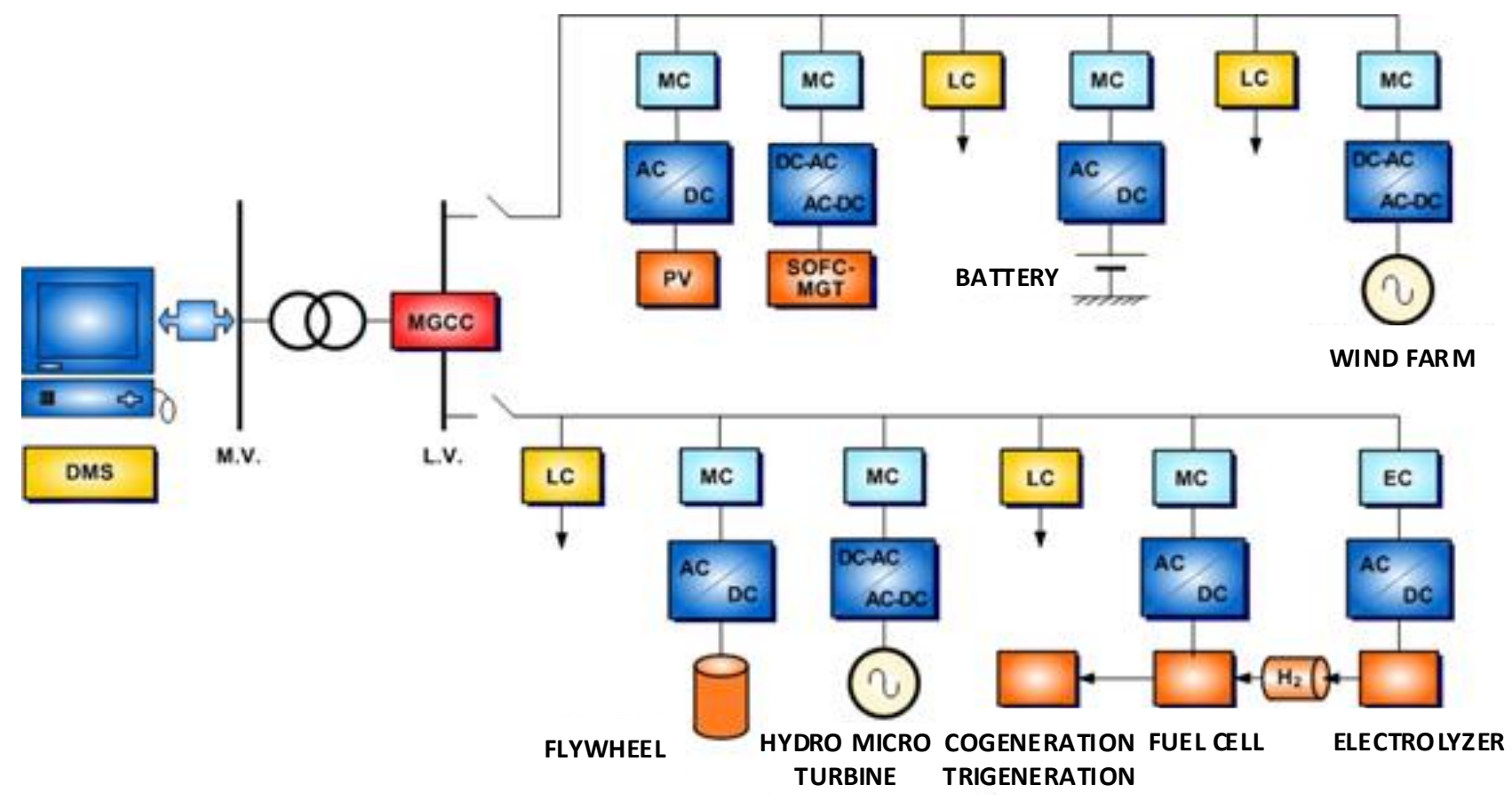

Fig. 1: Example of an electrical microgrid.

This primary controller acts as a local control for each DER unit and uses local measurements and responds to shortterm events, such as an island detection, sudden misalignments of active and / or reactive energy, and shared power. The secondary control operates in the time range from minutes to hours, and includes the discrete dispatch of DERs. This level is controlled by the MGCC. This controller is responsible for optimally coordinating and operating all the components connected in the same MG, ensuring the general maintenance of the grid parameters in both connected mode and island mode. The secondary control also incorporates strategies and operation controls such as intentional island operation, resynchronization and load-shedding. The tertiary control operates in the time range from hours to days and involves communication with the different MGCCs and the administration of the MG when it operates in the market. The main entities at this level are the distribution network operator (DNO) and the market operator (MO) who are delegates of the main network.

The MGs can be designed to operate in AC or DC. Each alternative has different characteristics, implying different advantages and drawbacks that must be assessed. The comparisons between the two types of MGs in terms of control, protections and power losses are analysed in [1720]. In these publications, the advantages and disadvantages of each technology are analysed, identifying

and classifying the configurations and feasible architectures required to implement a MG. The aforementioned objectives become a tool that allows choosing the most suitable MG that meets the specifications required in a given situation. In addition, this analysis constitutes an image of the state of the art of MGs, and also proposes research lines to address the current needs of the MGs and the further development of them.

\section{Generation technologies associated with electrical microgrids}

MGs are systems that integrate the resources of distributed generation to supply a variable number of distributed loads. Regarding distributed generators, they can be based on renewable or non-renewable resources. This characteristic allows to adequately exploit the available renewable resources in each location: water, wind, sun, biomass, etc.

Tables 1 and 2 indicate the most relevant properties of generation technologies that use renewable and nonrenewable resources [21]. These tables show that the systems based on wind energy and small hydroelectricity offer the highest efficiency among technologies based on renewable energies. Although ocean energy is a very promising source of energy, it still requires further research and development to be profitable and to reach the market with guarantees of success.

\section{Storage technologies associated with electrical microgrids}

The use of storage technologies improves the stability of the network, the quality and reliability of the power supply and the overall efficiency of a MG $[22,23]$. In Tables 3 and 4, the energy storage technologies available for most relevant $\mathrm{MG}$ applications are exposed [24, 25, 26]. It is interesting to note that the Superconducting Magnetic Energy Storage (SMES) provides high efficiency. However, this technology is still in the demonstration stage. 
Table 1: Main technologies of non-renewable DGs

\begin{tabular}{|c|c|c|c|c|c|c|c|}
\hline $\begin{array}{l}\text { Energy based } \\
\text { technology } \\
\text { type }\end{array}$ & Primary energy & $\begin{array}{l}\text { Output } \\
\text { type }\end{array}$ & $\begin{array}{l}\text { Module } \\
\text { power } \\
(\mathrm{kW})\end{array}$ & $\begin{array}{l}\text { Electrical } \\
\text { efficiency }\end{array}$ & $\begin{array}{l}\text { Overall } \\
\text { efficiency }\end{array}$ & Advantages & Disadvantages \\
\hline $\begin{array}{l}\text { Internal } \\
\text { combustion } \\
\text { engine }\end{array}$ & Diesel or gas & $\mathrm{AC}$ & $3-6,000$ & $30-43$ & $\sim 80-85$ & $\begin{array}{ll}\checkmark & \text { Low cost } \\
\checkmark & \text { High efficiency } \\
\checkmark & \text { Ability to use } \\
& \text { various inputs }\end{array}$ & \begin{tabular}{|ll}
$\mathbf{x}$ & Environmentally \\
& unfriendly \\
& emissions
\end{tabular} \\
\hline Gas turbine & Diesel or gas & $\mathrm{AC}$ & $\begin{array}{l}0.5- \\
30,000\end{array}$ & $21-40$ & $\sim 80-90$ & $\begin{array}{ll}\checkmark & \begin{array}{l}\text { High efficiencies } \\
\text { when using CHP }\end{array} \\
\checkmark & \begin{array}{l}\text { Environmentally } \\
\text { friendly }\end{array} \\
\checkmark & \text { Cost effective }\end{array}$ & \begin{tabular}{|ll}
$\mathbf{x}$ & $\begin{array}{l}\text { Too big for small } \\
\text { consumers }\end{array}$
\end{tabular} \\
\hline $\begin{array}{l}\text { Micro- } \\
\text { turbine }\end{array}$ & $\begin{array}{l}\text { Bio-gas, propane } \\
\text { or natural gas }\end{array}$ & $\mathrm{AC}$ & $30-1,000$ & $14-30$ & $\sim 80-85$ & $\begin{array}{ll}\checkmark & \text { Small size and light } \\
& \text { weight } \\
\checkmark & \begin{array}{l}\text { Easy start-up and } \\
\text { shut-down }\end{array} \\
\checkmark & \text { Low maintenance } \\
\text { costs }\end{array}$ & $\begin{array}{|ll|}\times & \text { Expensive } \\
& \text { technology } \\
\times & \text { Cost-effectiveness } \\
& \text { sensitive to the fuel } \\
& \text { price } \\
\times & \text { Environmentally } \\
& \text { unfriendly } \\
\text { emissions } \\
\end{array}$ \\
\hline Fuel cell & $\begin{array}{l}\text { Ethanol, } \mathrm{H}_{2}, \mathrm{~N}_{2}, \\
\text { natural gas, } \\
\text { PEM, DC } \\
\text { phosphoric acid } \\
\text { or propane }\end{array}$ & $\mathrm{DC}$ & $1-20,000$ & $5-55$ & $\sim 80-90$ & $\begin{array}{ll}\checkmark & \text { One of the most } \\
& \text { environmentally } \\
& \text { friendly generators } \\
\checkmark & \text { Extremely quiet } \\
\checkmark & \text { Useful for CHP } \\
& \text { applications } \\
\end{array}$ & $\begin{array}{|ll|}\mathbf{x} & \text { Extracting } \\
& \text { hydrogen is } \\
& \text { expensive } \\
\mathbf{x} & \text { Expensive } \\
\text { infrastructure for } \\
\text { hydrogen }\end{array}$ \\
\hline
\end{tabular}

Table 2: Main technologies of renewable DGs

\begin{tabular}{|c|c|c|c|c|c|c|c|}
\hline $\begin{array}{l}\text { Energy based } \\
\text { technology } \\
\text { type }\end{array}$ & $\begin{array}{l}\text { Primary } \\
\text { energy }\end{array}$ & $\begin{array}{l}\text { Output } \\
\text { type }\end{array}$ & $\begin{array}{l}\text { Module } \\
\text { power } \\
(\mathbf{k W})\end{array}$ & $\begin{array}{l}\text { Electrical } \\
\text { efficiency }\end{array}$ & $\begin{array}{l}\text { Overall } \\
\text { efficiency }\end{array}$ & Advantages & Disadvantages \\
\hline Wind & Wind & $\mathrm{AC}$ & $0.2-3,000$ & $-a$ & $\sim 50-80$ & \begin{tabular}{|ll}
$\checkmark$ & Day and night generation \\
$\checkmark$ & One of the most \\
& developed renewable \\
energy technologies
\end{tabular} & \begin{tabular}{|ll}
$\mathbf{x}$ & Still expensive \\
$\mathbf{x}$ & Storage \\
& $\begin{array}{l}\text { mechanisms } \\
\text { required }\end{array}$ \\
\end{tabular} \\
\hline $\begin{array}{l}\text { Photovoltaic } \\
\text { systems }\end{array}$ & Sun & $\overline{\mathrm{DC}}$ & $\begin{array}{l}0.02- \\
1,000\end{array}$ & $-a$ & $\sim 40-45$ & \begin{tabular}{|ll}
$\checkmark$ & Emission free \\
$\checkmark$ & Useful in a variety of \\
& applications
\end{tabular} & $\begin{array}{|ll|}x & \begin{array}{l}\text { Storage } \\
\text { mechanisms } \\
\text { required }\end{array}\end{array}$ \\
\hline $\begin{array}{l}\text { Biomass } \\
\text { gasification }\end{array}$ & Biomass & $\mathrm{AC}$ & $\begin{array}{l}100- \\
20,000\end{array}$ & $15-25$ & $\sim 60-75$ & \begin{tabular}{|ll}
$\checkmark$ & Minimal environmental \\
$\checkmark$ & impact \\
& Available throughout the \\
$\checkmark$ & world \\
& Alcohol and other fuels \\
produced by biomass are \\
efficient, viable, and \\
relatively clean burning
\end{tabular} & \begin{tabular}{|ll}
$\mathbf{x}$ & Still expensive \\
$\mathbf{x}$ & A net loss of energy \\
& in small scale
\end{tabular} \\
\hline $\begin{array}{l}\text { Small hydro } \\
\text { power }\end{array}$ & Water & $\mathrm{AC}$ & $\begin{array}{l}5- \\
100,000\end{array}$ & $-a$ & \begin{tabular}{|c|}
$\sim 90-98$ \\
\end{tabular} & \begin{tabular}{|ll}
$\checkmark$ & Economic and \\
& environmentally friendly \\
$\checkmark$ & Relatively low up-front \\
& investment costs and \\
& maintenance \\
$\checkmark$ & Useful for providing peak \\
& power and spinning
\end{tabular} & 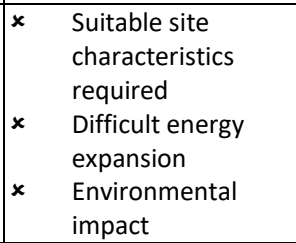 \\
\hline Geothermal & Hot water & $\mathrm{AC}$ & $\begin{array}{l}5000- \\
100,000\end{array}$ & $10-32$ & $\sim 35-50$ & \begin{tabular}{|ll}
$\checkmark$ & Extremely \\
& environmentally friendly \\
$\checkmark$ & Low running costs
\end{tabular} & $\begin{array}{ll}x & \begin{array}{l}\text { Non-availability of } \\
\text { geothermal spots in } \\
\text { the land of interest }\end{array}\end{array}$ \\
\hline Ocean energy & $\begin{array}{l}\text { Ocean } \\
\text { wave }\end{array}$ & $\mathrm{AC}$ & $10-1,000$ & $-a$ & $-a$ & \begin{tabular}{|ll} 
& High power density \\
$\checkmark$ & More predictable than \\
& solar or wind
\end{tabular} & \begin{tabular}{|ll}
$\mathbf{x}$ & Lack of commercial \\
& projects \\
$\mathbf{x}$ & Unknown \\
& operations and \\
& maintenance costs
\end{tabular} \\
\hline Solar thermal & $\begin{array}{l}\text { Sun and } \\
\text { power }\end{array}$ & $\mathrm{AC}$ & $\begin{array}{l}1,000- \\
80,000\end{array}$ & $30-40$ & $\sim 50-75$ & \begin{tabular}{|ll}
$\checkmark$ & Simple, low maintenance \\
$\checkmark$ & Operating costs nearly \\
& zero \\
$\checkmark$ & Mature technology
\end{tabular} & $\begin{array}{|ll|}\mathbf{x} & \text { Unknown } \\
& \text { operations and } \\
& \text { maintenance costs } \\
\mathbf{x} & \text { Low energy density } \\
\mathbf{x} & \text { Limited scalability }\end{array}$ \\
\hline
\end{tabular}

a: No data available. 
Table 3: Comparison of technical characteristics of EES systems.

\begin{tabular}{|c|c|c|c|c|c|c|c|}
\hline \multirow[t]{2}{*}{ Systems } & \multicolumn{2}{|c|}{ Power rating and discharge time } & \multicolumn{2}{|c|}{ Storage duration } & \multicolumn{3}{|l|}{ Capital cost } \\
\hline & Power rating & $\begin{array}{l}\text { Discharge } \\
\text { time }\end{array}$ & $\begin{array}{l}\text { Self-discharge } \\
\text { per day }\end{array}$ & $\begin{array}{l}\text { Suitable storage } \\
\text { duration }\end{array}$ & $\mathbf{\$} / \mathbf{k W}$ & $\mathbf{\$} / \mathbf{k W h}$ & c\$/kWh-Per cycle \\
\hline PHS & 100-5,000 MW & $1-24 \mathrm{~h}+$ & Very small & Hours-months & $600-2,000$ & $5-100$ & $0.1-1.4$ \\
\hline CAES & $5-300 \mathrm{MW}$ & $1-24 \mathrm{~h}+$ & Small & Hours-months & $400-800$ & $2-50$ & $2-4$ \\
\hline Lead-acid & $0-20 \mathrm{MW}$ & Second-hours & $0.1-0.3 \%$ & Minutes-days & $300-600$ & $200-400$ & $20-100$ \\
\hline NiCd & $0-40 \mathrm{MW}$ & Second-hours & $0.2-0.6 \%$ & Minutes-days & $500-1,500$ & $800-1,500$ & $20-100$ \\
\hline $\mathbf{N a S}$ & $50 \mathrm{~kW}-8 \mathrm{MW}$ & Second-hours & $\sim 20 \%$ & Seconds-hours & $1,000-3,000$ & $300-500$ & $8-20$ \\
\hline ZEBRA & $0-300 \mathrm{~kW}$ & Second-hours & $\sim 15 \%$ & Seconds-hours & $150-300$ & $100-200$ & $5-10$ \\
\hline Li-ion & $0-100 \mathrm{~kW}$ & Second-hours & $0.1-0.3 \%$ & Minutes-days & $1,200-4,000$ & $600-2,500$ & $15-100$ \\
\hline Fuel cells & $0-50 \mathrm{MW}$ & Seconds-24 h+ & Almost zero & Hours-months & $10,000+$ & & $6,000-20,000$ \\
\hline Metal-Air & $0-10 \mathrm{~kW}$ & Seconds-24 h+ & Very small & Hours-months & $100-250$ & $10-60$ & \\
\hline VRB & $30 \mathrm{~kW}-3 \mathrm{MW}$ & Seconds- $10 \mathrm{~h}$ & Small & Hours-months & $600-1,500$ & $150-1,000$ & $5-80$ \\
\hline $\mathrm{ZnBr}$ & $50 \mathrm{~kW}-2 \mathrm{MW}$ & Seconds-10 h & Small & Hours-months & $700-2,500$ & $150-1,000$ & $5-80$ \\
\hline PSB & $1-15 \mathrm{MW}$ & Seconds-10 h & Small & Hours-months & $700-2,500$ & $150-1,000$ & $5-80$ \\
\hline Solar fuel & $0-10 \mathrm{MW}$ & $1-24 \mathrm{~h}+$ & Almost zero & Hours-months & - & - & - \\
\hline SMES & $\begin{array}{l}100 \mathrm{~kW}-10 \\
\mathrm{MW}\end{array}$ & $\begin{array}{l}\text { Miliseconds-8 } \\
\mathrm{h}\end{array}$ & $10-15 \%$ & Minutes-hours & $200-300$ & $\begin{array}{l}1,000- \\
10,000\end{array}$ & \\
\hline Flywheel & $0-250 \mathrm{~kW}$ & $\begin{array}{l}\text { Miliseconds-15 } \\
\text { min }\end{array}$ & $100 \%$ & Seconds-minutes & $250-350$ & $1000-5000$ & $3-25$ \\
\hline Capacitor & $0-50 \mathrm{~kW}$ & $\begin{array}{l}\text { Miliseconds-60 } \\
\text { min }\end{array}$ & $40 \%$ & Seconds-hours & $200-400$ & $500-1,000$ & \\
\hline $\begin{array}{l}\text { Super- } \\
\text { capacitor }\end{array}$ & $0-300 \mathrm{~kW}$ & $\begin{array}{l}\text { Miliseconds-60 } \\
\text { min }\end{array}$ & $20-40 \%$ & Seconds-hours & $100-300$ & $300-2,000$ & $2-20$ \\
\hline Al-TES & $0-5 \mathrm{MW}$ & $1-8 \mathrm{~h}$ & $0.5 \%$ & Minutes-days & & $20-50$ & \\
\hline CES & $\begin{array}{l}100 \mathrm{~kW}-300 \\
\text { MW }\end{array}$ & $1-8 \mathrm{~h}$ & $0.5-1.0 \%$ & Minutes-days & $200-300$ & $3-30$ & $2-4$ \\
\hline HT-TES & $0-60 \mathrm{MW}$ & $1-24 \mathrm{~h}+$ & $0.05-1.0 \%$ & Minutes-months & & $30-60$ & \\
\hline
\end{tabular}

Table 4: Comparison of technical characteristics of EES systems

\begin{tabular}{|c|c|c|c|c|c|c|c|c|}
\hline \multirow[t]{2}{*}{ Systems } & \multicolumn{4}{|c|}{ Energy and power density } & \multicolumn{2}{|c|}{ Life time and cycle life } & \multicolumn{2}{|c|}{ Influence on environment } \\
\hline & Wh/kg & W/kg & Wh/L & W/L & $\begin{array}{l}\text { Life time } \\
\text { (years) }\end{array}$ & $\begin{array}{l}\text { Cycle life } \\
\text { (cycles) }\end{array}$ & Influence & Description \\
\hline PHS & $0.5-1.5$ & & $0.5-1.5$ & & $40-60$ & & Negative & $\begin{array}{l}\text { Destruction of trees and green } \\
\text { land for building the reservoirs }\end{array}$ \\
\hline CAES & $30-60$ & & $3-6$ & $0.5-2.0$ & $20-0$ & & Negative & $\begin{array}{l}\text { Emissions from combustion of } \\
\text { natural gas }\end{array}$ \\
\hline Lead-acid & $30-50$ & $75-300$ & $50-80$ & $10-400$ & $5-15$ & $500-1,000$ & Negative & Toxic remains \\
\hline NiCd & $50-75$ & $150-300$ & $60-150$ & & $10-20$ & $2,000-2,500$ & & \\
\hline $\mathrm{NaS}$ & $150-240$ & $150-230$ & $150-250$ & & $10-15$ & 2,500 & & \\
\hline ZEBRA & $100-120$ & $150-200$ & $150-180$ & $220-300$ & $10-14$ & $2,500+$ & & \\
\hline Li-ion & $75-200$ & $150-315$ & $200-500$ & & $5-15$ & $1,000-10,000+$ & & \\
\hline Fuel cells & $800-10,000$ & $500+$ & $\begin{array}{l}500- \\
3,000\end{array}$ & $500+$ & $5-15$ & $1,000+$ & Negative & $\begin{array}{l}\text { Remains and/or combustion of } \\
\text { fossil fuel }\end{array}$ \\
\hline Metal-Air & $150-3,000$ & & $\begin{array}{l}500- \\
10,000 \\
\end{array}$ & & & $100-300$ & Small & Little amount of remains \\
\hline VRB & $10-30$ & & $16-33$ & & $5-10$ & $12,000+$ & Negative & Toxic remains \\
\hline $\mathrm{ZnBr}$ & $30-50$ & & $30-60$ & & $5-10$ & $2,000+$ & & \\
\hline PSB & - & - & - & - & $10-15$ & & & \\
\hline Solar fuel & $\begin{array}{l}800- \\
100,000\end{array}$ & & $\begin{array}{l}500- \\
10,000 \\
\end{array}$ & & - & - & Benign & $\begin{array}{l}\text { Usage and storage of solar } \\
\text { energy }\end{array}$ \\
\hline SMES & $0.5-5$ & $\begin{array}{l}500- \\
2,000\end{array}$ & $0.2-2.5$ & $\begin{array}{l}1,000- \\
4,000\end{array}$ & $20+$ & $100,000+$ & Negative & Strong magnetic fields \\
\hline Flywheel & $10-30$ & $\begin{array}{l}400- \\
1,500\end{array}$ & $20-80$ & $\begin{array}{l}1,000- \\
2,000\end{array}$ & $\sim 15$ & $20,000+$ & $\begin{array}{l}\text { Almost } \\
\text { none }\end{array}$ & \\
\hline Capacitor & $0.05-5$ & $100,000+$ & $2-10$ & $100,000+$ & $\sim 5$ & $50,000+$ & Small & Little amount of remains \\
\hline $\begin{array}{l}\text { Super- } \\
\text { capacitor }\end{array}$ & $2.5-15$ & $\begin{array}{l}500- \\
5,000\end{array}$ & & $100,000+$ & $20+$ & $100,000+$ & Small & Little amount of remains \\
\hline Al-TES & $80-120$ & & $80-120$ & & $10-20$ & & Small & \\
\hline CES & $150-250$ & $10-30$ & $120-200$ & & $20-40$ & & Positive & $\begin{array}{l}\text { Removing contaminates during } \\
\text { air liquefaction (Charge) }\end{array}$ \\
\hline HT-TES & $80-200$ & & $120-500$ & & $5-15$ & & Small & \\
\hline
\end{tabular}

Table 3 also shows that there are other technologies such as Pumping Hydraulic Storage (PHS) or Compressed Air Energy Storage Systems (CAES) that, despite their lower efficiencies, have higher capacities with longer lifetimes. The connection schemes of the most used storage technologies can be found in [27]. These technologies must ensure a secure energy supply and must help achieving better energy quality in MGs.

\section{Microgrids worldwide \\ currently}

operating

In this section, structures of electric MGs that have been developed worldwide are exposed, highlighting the most important properties of them. In this sense, several installations of electrical MGs deployed worldwide have 
been considered to analyse their results. Tables 5, 6, 7 and 8 summarize the experiences in MGs classified according to their location, resources, storage technologies, controls, loads, etc. [15]. Some organizations are carrying out studies on MGs, such as the Consortium for Electric Reliability Technology Solutions (CERTS) in the USA. The Consortium for Electric Reliability Technology Solutions (NEDO) in Japan, and MICROGRIDS and MORE MICROGRIDS in Europe. Most of the MGs implemented have at least one of the following purposes:

1) Give access to electricity to remote areas, where it is difficult to connect to the main grid. Some examples could be: the micro-networks in Africa (Akkan, Diakha Medina, Lucingweni [28, 29, 30] and remote communities that operate in islanded mode (farm in Kozuf, Macedonia [31]). Furthermore, islanded mode lets the islands to be autonomous when accessing to electricity.
2) Development of studies: many projects use their own micro-networks to study control schemes, communication protocols, $\mathrm{P} / \mathrm{f}$ and $\mathrm{Q} / \mathrm{V}$ controls, such as the MORE MICROGRIDS project [31] or the DISPOWER project [32]. In addition, many universities [15] stand out, such as Manchester, Leuven, Santa Clara, San Diego [33], Howard, Hefei University, and Technological Institutes, such as the Illinois Institute of Technology [34], which has developed its own micro-network to carry out experiments being also self-producers.

3) Improve security in case of wars or disasters: 40 military bases in the USA are operating as MGs and the Department of Defense is researching the deployment of small MGs in problematic areas [148]. This interest in MGs has grown in the USA after hurricanes like Katrina and Sandy, which caused long periods without energy.

Table 5: Examples of microgrids in Europe.

\begin{tabular}{|c|c|c|c|c|c|c|c|c|}
\hline \multicolumn{2}{|l|}{ Situation } & \multirow[t]{2}{*}{ Project manager } & \multicolumn{2}{|l|}{ Type } & \multicolumn{2}{|c|}{ Control } & \multicolumn{2}{|c|}{ Structure } \\
\hline Place & Country & & Real & $\begin{array}{l}\text { Test- } \\
\text { bed }\end{array}$ & Cent. & Decent. & $\mathbf{A C}$ & DC \\
\hline Bornholm island & Denmark & More microgrids project & $\checkmark$ & & $\mathrm{a}$ & & $\checkmark$ & \\
\hline Lyon & France & NEDO & & $\checkmark$ & $\mathrm{a}$ & & $\checkmark$ & \\
\hline Kassel & Germany & $\begin{array}{l}\text { The Institut für Solare Energieversorgungstechnik } \\
\text { (ISET), } \\
\text { University of Kassel Institute for Electrical Energy } \\
\text { Technology (IEE) }\end{array}$ & & $\checkmark$ & $\checkmark$ & & $\checkmark$ & \\
\hline $\begin{array}{l}\text { Manheim } \\
\text { Wallstadt }\end{array}$ & Germany & More microgrids project & $\checkmark$ & & & $\checkmark$ & & \\
\hline Stutensee & Germany & DISPOWER project & $\checkmark$ & & $\checkmark$ & & $\checkmark$ & \\
\hline Atenas & Greece & National Tchnical University of Athens (NTUA) & & $\checkmark$ & & $\checkmark$ & $\checkmark$ & \\
\hline Milan & Italy & Ricerca Sistema Energetico (RSE) & & $\checkmark$ & $\checkmark$ & & & $\checkmark$ \\
\hline Agria pig farm & Macedonia & More microgrids project & $\checkmark$ & & $\mathrm{a}$ & & $\checkmark$ & \\
\hline Bronsbergen & Netherlands & More microgrids project & $\checkmark$ & & $\checkmark$ & & $\checkmark$ & \\
\hline Groningen & Netherlands & KEMA & $\checkmark$ & & & $\checkmark$ & $\checkmark$ & \\
\hline Utsira & Norway & StatoilHydro and Enercon & $\checkmark$ & & $\checkmark$ & & $\checkmark$ & \\
\hline Ilhavo & Portugal & More microgrids project & $\checkmark$ & & $\checkmark$ & & $\checkmark$ & \\
\hline Barcelona & Spain & Institut de Recerca en Energia de Catalunya (IREC) & & $\checkmark$ & $\mathrm{a}$ & & $\checkmark$ & \\
\hline Derio & Spain & More microgrids project & & $\checkmark$ & $\checkmark$ & $\checkmark$ & $\checkmark$ & \\
\hline Miñano & Spain & Ikerlan & & $\checkmark$ & $\checkmark$ & & $\checkmark$ & \\
\hline $\begin{array}{l}\text { Horizon, } \\
\text { Manchester }\end{array}$ & UK & H2Ope & $\checkmark$ & & a & & $\mathrm{a}$ & \\
\hline Manchester & UK & University of Manchester & $\checkmark$ & & $\checkmark$ & & $\checkmark$ & \\
\hline
\end{tabular}

a: No data found.

Table 6: Examples of microgrids in Asia and Oceania.

\begin{tabular}{|c|c|c|c|c|c|c|c|c|}
\hline \multicolumn{2}{|l|}{ Situation } & \multirow[t]{2}{*}{ Project manager } & \multicolumn{2}{|l|}{ Type } & \multicolumn{2}{|c|}{ Control } & \multicolumn{2}{|c|}{ Structure } \\
\hline Place & Country & & Real & Test-bed & Cent. & Decent. & $\mathbf{A C}$ & DC \\
\hline Newcastle & Australia & CSIRO Energy Center & & $\checkmark$ & $\checkmark$ & & $\checkmark$ & \\
\hline Hefei & China & Hefei University of Technology (HFUT) & & $\checkmark$ & & $\checkmark$ & & \\
\hline Tianjin & China & Tianjin University & & $\checkmark$ & $\checkmark$ & & $\checkmark$ & \\
\hline Changwon & Korea & Korea Electrotechnology Research Institute (KERI) & & $\checkmark$ & $\checkmark$ & & $\checkmark$ & \\
\hline Uttar Pradesh & India & Mera Gao Power (MGP) & & $\checkmark$ & $\checkmark$ & & $\checkmark$ & \\
\hline Aichi & Japan & Aichi Institute of Technology (AIT), NEDO & $\checkmark$ & & & & & \\
\hline Akagi & Japan & NEDO & $\checkmark$ & $\checkmark$ & & $\checkmark$ & & \\
\hline Hachinoche & Japan & NEDO & $\checkmark$ & & $\checkmark$ & & $\checkmark$ & \\
\hline Kyoto Eco-Energy & Japan & NEDO & & $\checkmark$ & $\checkmark$ & & $\checkmark$ & \\
\hline Sendai & Japan & NEDO & $\checkmark$ & & $\checkmark$ & $\checkmark$ & & \\
\hline
\end{tabular}

Table 7: Examples of microgrids in North America.

\begin{tabular}{|c|c|c|c|c|c|c|c|c|}
\hline \multicolumn{2}{|l|}{ Situation } & \multirow[t]{2}{*}{ Project manager } & \multicolumn{2}{|l|}{ Type } & \multicolumn{2}{|c|}{ Control } & \multicolumn{2}{|c|}{ Structure } \\
\hline Place & Country & & Real & $\begin{array}{l}\text { Test- } \\
\text { bed }\end{array}$ & Cent. & Decent. & $\mathbf{A C}$ & DC \\
\hline Boston Bar & Canada & BC Hydro & $\checkmark$ & & & $\checkmark$ & $\checkmark$ & \\
\hline Senneterre & Canada & Hydro Quebec (HQ) & $\checkmark$ & & & & $\checkmark$ & \\
\hline $\begin{array}{l}\text { Albuquerque, New } \\
\text { Mexico }\end{array}$ & USA & $\begin{array}{l}\text { NEDO, Sandia National Laboratories, The University of } \\
\text { New Mexico and Japanese companies }\end{array}$ & & $\checkmark$ & $\mathrm{a}$ & & $\mathrm{a}$ & \\
\hline
\end{tabular}




\begin{tabular}{|c|c|c|c|c|c|c|}
\hline Ansonia, Connecticut & USA & $\begin{array}{l}\text { Pareto Energy, Ltd. And Connecticut Center for } \\
\text { Advanced Technology (CCAT) }\end{array}$ & $\checkmark$ & & $\mathrm{a}$ & $\mathrm{a}$ \\
\hline $\begin{array}{l}\text { Borrego Springs, } \\
\text { California }\end{array}$ & USA & San Diego Gas \& Electric Company (SDG \& E) & $\checkmark$ & & $\checkmark$ & $\checkmark$ \\
\hline Columbus & USA & Dolan Technology Center & & $\checkmark$ & & $\checkmark$ \\
\hline Washington & USA & Howard University & & $\checkmark$ & $\mathrm{a}$ & $\mathrm{a}$ \\
\hline Chicago & USA & Illinois Institute of Technology & & $\checkmark$ & & $\mathrm{a}$ \\
\hline $\begin{array}{l}\text { Los Alamos, New } \\
\text { Mexico }\end{array}$ & USA & NEDO & & $\checkmark$ & $\mathrm{a}$ & $\mathrm{a}$ \\
\hline Madison & USA & University of Wisconsin & & $\checkmark$ & & $\checkmark$ \\
\hline $\begin{array}{l}\text { Marin County, } \\
\text { California }\end{array}$ & USA & Xanthus Consulting International, Infotility, Inc. & $\checkmark$ & & & $\checkmark$ \\
\hline California & USA & Santa Clara University & & $\checkmark$ & $\mathrm{a}$ & $\checkmark$ \\
\hline $\begin{array}{l}\text { Stamford, } \\
\text { Connecticut }\end{array}$ & USA & Pareto Energy & $\checkmark$ & & $\mathrm{a}$ & $\mathrm{a}$ \\
\hline San Diego & USA & University California San Diego & & $\checkmark$ & $\mathrm{a}$ & $\mathrm{a}$ \\
\hline $\begin{array}{l}\text { Twenty-nine palms, } \\
\text { California }\end{array}$ & USA & General Electric (GE) & $\checkmark$ & & $\checkmark$ & $\checkmark$ \\
\hline
\end{tabular}

a: No data found.

Table 8: Examples of islanded microgrids all around the world.

\begin{tabular}{|c|c|c|c|c|c|c|c|}
\hline \multicolumn{3}{|l|}{ Situation } & \multirow[t]{2}{*}{ Project manager } & \multicolumn{2}{|c|}{ Control } & \multicolumn{2}{|c|}{ Structure } \\
\hline Region & Place & Country & & Cent. & Decent. & $\mathbf{A C}$ & DC \\
\hline \multirow[t]{3}{*}{ Africa } & Akkan & Morocco & 2 & $\checkmark$ & & $\checkmark$ & \\
\hline & Diaka Madina & Senegal & 2 & $\checkmark$ & & $\checkmark$ & \\
\hline & Lucingweni & South Africa & $\begin{array}{l}\text { National Energy Regulator of South Africa } \\
\text { (NERSA) }\end{array}$ & & $\checkmark$ & $\checkmark$ & $\checkmark$ \\
\hline Antartic & Princess Elisabeth Station & Antartic & Laborelec & $\checkmark$ & & $\checkmark$ & \\
\hline \multirow{3}{*}{ Asia } & Kuroshima island & Japan & Kyushu Electric Power & $\checkmark$ & & $\checkmark$ & \\
\hline & Miyako island, Okinawa & Japan & Okinawa Electric Power Company (OEPC) & $\mathrm{a}$ & & $\checkmark$ & \\
\hline & Town Island & Hong Kong & Hong Kong University (HKU) & $\checkmark$ & & $\checkmark$ & \\
\hline Europe & Kythnos & Greece & & $\checkmark$ & & $\checkmark$ & \\
\hline \multirow{7}{*}{$\begin{array}{l}\text { North } \\
\text { America }\end{array}$} & Bella Cola & Canada & BC Hydro, GE, PowerTech & $\checkmark$ & & $\checkmark$ & \\
\hline & Hartley Bay & Canada & Pulse Energy (ICE) & & $\checkmark$ & $\checkmark$ & \\
\hline & Kasabonika Lake & Canada & Hydro One, GE, University of Waterloo & $\checkmark$ & & $\checkmark$ & \\
\hline & Nemiah Valley & Canada & NRCan & $\checkmark$ & & $\checkmark$ & \\
\hline & Ramea Island & Canada & $\begin{array}{l}\text { N \& L Hydro, Nalcor Energy, NRCan, Frontier } \\
\text { Power }\end{array}$ & $\checkmark$ & & $\checkmark$ & \\
\hline & Colonias, Texas & USA & $\begin{array}{l}\text { Texas State Energy Conservation Office (SECO), } \\
\text { Texas Engineering Experiment Station, Xtreme } \\
\text { Power }\end{array}$ & $\checkmark$ & & $\checkmark$ & \\
\hline & $\begin{array}{l}\text { Fort Bragg, North } \\
\text { Caroline }\end{array}$ & USA & Encorp, Honeywell & $\checkmark$ & & $\checkmark$ & \\
\hline Oceania & Kings Canyon & Australia & UNSW (Sydney) & $\checkmark$ & & $\checkmark$ & \\
\hline \multirow{2}{*}{$\begin{array}{l}\text { South } \\
\text { America }\end{array}$} & Chico Mendes & Brazil & Electrobas & $\checkmark$ & & $\checkmark$ & $\checkmark$ \\
\hline & Ilha da Ferradura & Brazil & $\mathrm{a}$ & $\checkmark$ & & $\checkmark$ & \\
\hline
\end{tabular}

a: No data found.

\section{Conclusion}

This paper contributes to the definition of the electrical MGs usage in power systems. In this sense, MGs provide an adequate structure in terms of performance of a distributed energy generation system. The MGCC links, controls and manages smartly this type of generation systems.

A review of most relevant properties in energy generation and storage technologies in the development of electric MGs has been addressed. In this sense, installed capacity, response time, power range, operating modes, efficiencies, electronic devices associated to its integration, etc. are analysed.

Furthermore, major drawbacks for the integration of MGs in power system are exposed. Additionally, technical characteristics of several MGs currently being analysed are specified, among further research proposals from public and private institutions worldwide.

\section{Acknowledgement}

The authors thank the support from the Gipuzkoa Provincial Council (project Etorkizuna Eraikiz 2019 DGE19/03), the Basque Government (GISEL research group IT1083-16), as well as from the University of the Basque Country UPV/EHU (PES16/31 and PES17/08).

\section{References}

[1] Green M. Community power. Nat Energy 2016;1:16014. http://dx.doi.org/10.1038/nenergy.2016.14.

[2] A. Hirsch, Y.Parag, J. Guerrero, «Microgrids: A review of technologies, key drivers, and outstanding issues», Vol. 90, pp. 402-411, 2018.

[3] P. Barker, B. Johnson, A. Maitra. «Investigation of the technical and economic feasibility of micro-grid based power systems». Palo Alto, CA. EPRI; 2001.

[4] A.B. Lovins, Rocky Mountain Instituteeditors. Small is profitable. 1st ed.Snowmass, CO: Rocky Mountain Institute; 2002. 
[5] H. Lotfi, A. Khodaei, «Hybrid AC / DC microgrid planning» IEEE Trans Power Syst, Vol. 8, pp. 296-304, 2016

[6] A.Gururani, S.R. Mohanty, J.C.Mohanta. «Microgrid protection using Hilbert-Huang transform based-differential scheme», IET Gener Transm DistribVol. 10, pp. 3707-3716, 2016. [7] D.P. Mishra, S.R. Samantaray, G. Joos, «A combined wavelet and data-mining based intelligent protection scheme for microgrid», IEEE Trans Smart Grid, Vol.7, pp. 2295-2304, 2016. [8] M.H.Cintuglu, T. Ma, O.A. Mohammed, «Protection of autonomous microgrids using agent-based distributed communication», IEEE Trans Power Deliv, Vol.32, pp. 351-360, 2016.

[9] K. Lai, M.S. Illindala, M.A.Haj-Ahmed, «Comprehensive protection strategy for an islanded microgrid using intelligent relays», IEEE Trans Ind Appl, Vol.8, pp. 47-55, 2016.

[10] E. Ragaini, E. Tironi , S. Grillo, L. Piegari, M. Carminati, «Ground fault analysis of low voltage DC micro-grids with active front-end converter», Proceedings of the 3rd Renew. Power Gener. Conference, Naples, Italy, p. 1-6, 2014.

[11] I. Patrao, E. Figueres, G. Garcerá, R. González-Medina, «Microgrid architectures for low voltage distributed generation. Renew Sustain Energy Rev», Vol.43 pp.415-424, 2015

[12] K. S. Rajesh, S.S. Dash, R. Rajagopal, R. Sridhar, «A review on control of ac microgrid», Renew Sustain Energy Rev Vol. 71, pp. 814- 819, 2017.

[13] O.Palizban, K. Kauhaniemi, J.M. Guerrero, «Microgrids in active network management- part II: system operation, power quality and protection», Rene Sustain Energy Rev, Vol. 36, pp. 440-451, 2014

[14] D. Olivares, A. Mehrizi-Sani, A. Etemadi, C. Canizares, R. Iravani , M. Kazerani, A. Hajimiragha , O. Gomis-Bellmun, M. Saeedifard , R. Palma-Behnke , G. Jimenez-Estevez , N. Hatziargyriou , «Trends in microgrid control» IEEE Trans Smart Grid, Vol.5, pp. 1905-1919, 2014.

[15] E. Planas, A. Gil-de-Muro, J. Andreu,I. Kortabarria, I. Martínez de Alegría, «General aspects, hierarchical controls and droop methods in microgrids: a review», Renew Sustain Energy Rev, Vol. 17, pp. 147-159, 2013.

[16] S. Marzal, R. Salas, R. González-Medina, G. Garcerá, E. Figueres, «Current challenges and future trends in the field of communication architectures for microgrids», Renew Sustain Energy Rev Vol. 82, pp. 3610-3622, 2018.

[17] R. Asad, A. Kazemi, «A quantitative analysis of effects of transition from ac to dc system, on storage and distribution systems: smart grid technologies», In: IEEE PES Asia-Pacific power and energy engineering conference (APPEEC), pp. 1-5, 2012.

[18] J. Guerrero, P.C.Loh, T.L. Lee, M. Chandorkar, «Advanced control architectures for intelligent microgrids-part II: power quality, energy storage, and ac/dc microgrids», IEEE Trans Ind Electron, Vol. 60, pp.1263-1270, 2013.

[19] S. Ali, M. Babar, S. Maqbool, E. Al-Ammar, «Comparative analysis of ac de microgrids for the saudi arabian distribution system», In: IEEE/PES transmission and distribution conference and exposition (T\&D), pp. 1-8, 2012.

[20] J.J. Just, F. Mwasilu, J. Lee, J. Jung, «AC-microgrids versus DC-microgrids with distributed energy resources: a review», Renew Sustain Energy Rev, Vol. 24 pp.387-405, 2013.

[21] E. Planas, J. Andreu, J.I. Gárate, I. Martínez de Alegría, E. Ibarra, «AC and DC technology in microgrids: A review», Renew Sustain Energy Rev, Vol. 43, pp. 726-749, 2015.

[22] P. Ribeiro, B.Johnson, M. Crow, A.Arsoy, Y. Liu, «Energy storage systems for advanced power applications» In: Proceedings of the IEEE, Vol. 89, pp. 1744-1756, 2001.

[23] B. Kroposki, R. Lasseter, T. Ise, S. Morozumi, S. Papatlianassiou, N.Hatziargyriou, «Making microgrids work», IEEE Power Energy Mag, Vol 6, pp. 40-53, 2008.

[24] T. Kousksou, P. Bruel, A.Jamil, T.E.Rhafiki, Y. Zeraouli, «Energy storage: applications and challenges», Solar Energy Materials and Solar Cells, Vol. 120, pp. 59-80, 2014.
[25] H.L.Ferreira, R.Garde, G. Fulli, W. Kling, J.P. Lopes, «Characterisation of electrical energy storage technologies», Energy, Vol. 53, pp. 288-98. 2013

[26] H. Chen, T.N. Cong, W. Yang, C. Tan, Y. Li, Y. Ding, «Progress in electrical energy storage system: a critical review», Progr Nat Sci , Vol. 19, pp.291-312., 2009

[27] J. Kaldellis, D. Zafirakis, K. Kavadias, «Techno-economic comparison of energy storage systems for island autonomous electrical networks», Renew Sustain Energy Rev, Vol. 13, pp.378-392, 2009

[28] F.Martin-Martínez, A. Sánchez-Miralles, M. Rivier, «A literature review of Microgrids: A functional layer based classification», Renew Sustain Energy Rev, Vol. 62, pp. 11331153,2016

[29] A.Graillot, TTA: Portfolio African projects PV hybrid micro-grids; 2012.

[30] S. Mulaudzi, N. Qase N, New and renewable energy: minigrid hybrid viability and replication potential: the Hluleka and Lucingweni pilot projects, Dep Miner Energy Pretoria. New Renew Energy, 2008.

[31] More microgrids project, microgrids project. www.microgrids.eu n.d. 〈http://www.microgrids.eu/default.php〉. [32] N.W.A.Lidula, A.D. Rajapakse, «Microgrids research: a review of experimental microgrids and test systems», Renew Sustain Energy Rev, Vol.15, pp.186-202, 2011

[33] Y. Agarwal, T. Weng, R.K. Gupta, «Understanding the role of buildings in a Smart microgrid», In: proceedings of the design, automation and test in Europe conference and exhibition, Grenoble: IEEE, pp.1-6, 2011

[34] Microgrid at Illinois Institute of Technology. Microgrid at Illinois Institute of Technology n.d 〈http://www.iitmicrogrid.net/〉

[35] Navigant Research. Military Microgrids n.d. 〈http://www.navigantresearch.com/webinar/militarymicrogrids- 2$\rangle$ 